\title{
The effect of a professional cricket match schedule on selected immune parameters
}

\author{
A W Williams (MB ChB, MPhil (Exercise Science) \\ K H Myburgh (PhD (Physiology)) \\ C Smith (B Tech (Biomedical Technology), MPhil (Exercise Science)) \\ Department Physiological Sciences, University of Stellenbosch, South Africa
}

\begin{abstract}
Objective. The impact of a professional cricket match schedule on white blood cell (WBC) distribution and lymphocyte activation (CD69 expression) was investigated.

Methods. After a 3-month pre-season training period, physical and immune parameters were determined in 14 male cricketers before (B) and after (A) an intensive 5week match schedule.

Results. Exercise test results were unchanged from $B$ to A. Total WBC counts were similar, but total lymphocyte and lymphocyte subpopulation counts decreased significantly. The CD4:CD8 ratio did not change. After in vitro stimulation, percentage CD4+CD69+ cells increased (B: $54.4 \pm 9.7 \%, \mathrm{~A}: 64.0 \pm 8.5 \%, p<0.01)$, but absolute CD4+CD69+ cell counts did not change from B to A. In contrast, both the \%CD8+CD69+ cells and absolute CD8+CD69+ cell count remained similar.

Conclusion. A strenuous, interregional, professional cricket match schedule resulted in a decreased number of lymphocytes, but relatively increased in vitro reactivity of CD4+ cells, thus maintaining the absolute capacity of the CD4+ cells to become activated on stimulation. In cricketers who suffered upper respiratory tract symptoms during the match schedule $(N=7)$, none of the immune parameters investigated differed significantly from the others at $\mathrm{B}$ or $\mathrm{A}$.
\end{abstract}

\section{CORRESPONDENCE:}

\section{Smith}

Department of Physiological Sciences

University of Stellenbosch

Private Bag X1

Matieland

7602

Tel: 021-808 4388

Fax: 021-808 3145

E-mail: csmith@sun.ac.za

\section{Introduction}

Considerable physical and mental demands are placed on professional team sport players, with competitive events becoming more frequent and training schedules increasingly intense. In addition to exercise stress, a number of other stressors such as lack of sleep, mental stress, possible allergies to pollen or grass, frequent travel and prolonged exposure to ultraviolet (UV) radiation, also add to the demands of modern-day cricket.

Studies have shown that, in general, excessive exercise during training and competition leads to immunosuppression, or possibly a shift from cell-mediated to humoral immunity, especially in endurance-trained athletes. ${ }^{22,31,34}$ Epidemiological studies on endurance athletes participating in long duration, competitive events ${ }^{2,13,21,23,31}$ have shown an increased incidence of upper respiratory tract symptoms (URTS). Although an earlier epidemiological survey of ultramarathon runners reported increased URTS in faster runners, ${ }^{23}$ a more recent laboratory study of trained cyclists showed that in less extreme sports events, exercise duration has a greater suppressive effect on selected parameters of immune system function than exercise intensity. ${ }^{26}$

To understand these results more fully, it is necessary to consider findings of research at cellular level. Studies have indicated that an acute bout of aerobic exercise decreased CD4:CD8 ratio during exercise, with a subsequent transient increase above baseline, post-exercise. ${ }^{17,27,30,33}$ However, strenuous training and competition over time may lead to chronically decreased CD4:CD8 ratios in athletes. ${ }^{1}$ Specifically, Baj et al. ${ }^{1}$ reported a significantly decreased CD4+ cell count accounting for the decrease in CD4:CD8 ratio in competitive cyclists after a 6-month season. This suggests a shift from specific immunity (CD4 cells) to nonspecific immunity (CD8 cytotoxic cells), or suppression of certain B- and T-cell mediated immune functions in these athletes.

Lymphocytes expressing the cluster differentiation markers CD4 (helper T), CD8 (suppressor/cytotoxic T), CD19 (B) or CD15/56 (natural killer (NK)) have undergone primary proliferation during the haematopoietic process and are usually in a quiescent state, and these CD markers may be used to identify the specific lymphocyte subpopulations. In response to mitogen exposure some of these cells will become activated and capable of secondary proliferation, which is vital for proper immune function. Cells committed to secondary proliferation express CD69, ${ }^{14}$ which has been linked to their activation, proliferation and cytotoxic function. ${ }^{4,5,35}$ The wide cel- 
lular distribution of CD69 and its association with intracellular signal transduction ${ }^{8}$ suggest a central role for its expression in immune system function.

Few studies ${ }^{10,12,27}$ have investigated exercise-induced modulation of CD69 expression. Ronsen et al. ${ }^{27}$ reported that 1 bout of cycling (65 minutes at $75 \%$ maximal oxygen uptake $\left.\left(\mathrm{VO}_{2 \max }\right)\right)$ did not significantly affect mitogen-induced CD69 expression in CD4+, CD8+ or NK cells. However, after 2 bouts of cycling separated by 3 hours, no effect was evident in the CD69 expression of CD4+ or CD8+ cells, but percentage of CD69+ NK cells was transiently decreased for 4 hours after the second bout. Similarly, Green et al. ${ }^{12}$ reported no change in the ability of CD4+ or CD8+ cells to become activated or to proliferate after a single 1-hour bout of treadmill running at $95 \%$ of ventilatory threshold. These study designs did not allow an assessment of the chronic effect of training in these elite athletes. In summary, the main conclusion that can be drawn from available research is that endurance exercise without sufficient rest between bouts seems to have only a transient inhibiting effect on NK cell activation and that chronic exercise training may cause a shift toward mobilisation of cytotoxic cell populations. ${ }^{18,19}$

However, monitoring of elite swimmers over 12 weeks of training ${ }^{11}$ showed no association between NK cell count and URTI, suggesting that the humoral immune response may have a more significant role in protection from infection over a whole season. The longer-term effect of several weeks of strenuous exercise on CD69 expression in CD4+ and CD8+ cells has not yet been established in any exercise model. Since activation and proliferation of lymphocytes are crucial for mounting an immune response against pathogens, it is important for athletes to understand the influence of their sport on the immune system, to assist in the prevention of illness.

In competitive athletes exercise is not the only stressor, and effects of other possible additional stressors should be considered. For example, UV-B radiation has been shown to decrease lymphocyte function in in vitro culture studies, ${ }^{16}$ and to increase DNA damage in mononuclear cells. ${ }^{15}$ Also, lack of sleep, mental stress and frequent travel, which includes the risk of exposure to novel pathogens, have been named as additional factors which amplify the risk of infection in athletes. ${ }^{20}$ Long-distance air travel has been reported to impair T-cell dependent cellular immunity, ${ }^{28,29}$ possibly due to cortisol-induced down-regulation of T-helper 2 cells. ${ }^{29}$
To begin to profile the cumulative effect of a variety of stressors, we studied athletes participating in a sport with frequent, long-duration match-play, with intermittent bursts of exercise, who also had a frequent schedule of matches, some requiring air travel. Specifically, the effect of an intermittent, strenuous cricket match-play schedule lasting 5 weeks on lymphocyte subpopulation distribution and the expression of CD69 in CD4+ and CD8+ T-cells after in vitro stimulation was determined. A second purpose was to investigate whether these immune parameters (determined before and after the match-schedule) were associated with either the fitness performance of the subjects at the same time points, or URTS experienced during the 5 weeks.

\section{Methods}

\section{Subjects}

Fourteen healthy, fit male cricketers (mean age $25.4 \pm 4.8$ years) from a regional professional cricket team completed the study ( 3 other recruited players were excluded due to injury sustained during the study). The subject group included 10 batsmen, 1 wicket-keeper-batsman, 5 fast bowlers and 1 spin-bowler batsman, thereby representing all positions in a cricket team. All subjects had been participating in cricket for more than 10 years, and had been playing professional cricket for an average of $4.9 \pm 3.3$ years. Ethical approval was obtained from the University of Stellenbosch before subjects gave voluntary, written, informed consent. Initially, all subjects completed a health history questionnaire and underwent a general medical examination before the study. No subject reported a history of allergic reactions to grass or pollen, or of asthma. No pharmacological treatment was administered to any subject in the month prior to the study.

\section{Exercise schedule}

The competitive season used as intervention in this study lasted 5 weeks and consisted of five 4-day provincial cricket matches with air travel (matches were played at venues $\sim 2000 \mathrm{~km}$ apart) and training sessions in between. A detailed description of exercise and training (volume and intensity) prescribed before and during the study period is given in Table I. These schedules were prescribed and supervised by a professional biokineticist appointed by the team management. Subjects kept a daily diary of health (including the following symptoms of URTS: sore throat, run-

\begin{tabular}{|c|c|c|c|c|c|}
\hline Phase & $\begin{array}{l}\text { Sessions } \\
\text { per week }\end{array}$ & $\begin{array}{l}\text { Duration of } \\
\text { session (hrs) }\end{array}$ & $\begin{array}{l}\text { Total duration of } \\
\text { sessions (hrs) }\end{array}$ & $\begin{array}{c}\% \text { aerobic } \\
\text { exercise }\end{array}$ & $\begin{array}{c}\% \text { anaerobic } \\
\text { exercise }\end{array}$ \\
\hline \multicolumn{6}{|l|}{ Pre-season: } \\
\hline Gymnasium session & 2 & 2 & 4 & 50 & 50 \\
\hline Cricket training session & 3 & 4 & 12 & 70 & 30 \\
\hline Cricket match (regional) & 1 & 6 (intermittent) & 6 (intermittent) & $50-70$ & $30-50$ \\
\hline \multicolumn{6}{|l|}{ In-season } \\
\hline Gymnasium session & 1 & 3 & 3 & 100 & 0 \\
\hline Cricket training session & 2 & 4 & 8 & 70 & 30 \\
\hline Cricket match (provincial) & 4 & 6 (intermittent) & 24 (intermittent) & $50-70$ & $30-50$ \\
\hline
\end{tabular}


ning nose, cough, sinus symptoms, headaches), intake of supplements and medication and reported to the team doctor on a weekly basis. These subjects were professional athletes and were used to reporting all health-related issues to the team doctor without delay. Athletes participated in both home and away games and were therefore accommodated together and in their home environments during the month of the study. Whether or not any family members had URTS symptoms during the study was not recorded.

\section{Fitness tests and blood sampling}

All measures were taken at two time points: before $(B)$ and after $(\mathrm{A})$ the 5-week schedule. $\mathrm{VO}_{2 \max }$ was estimated using an incremental $20 \mathrm{~m}$ shuttle run (Bleep Test). ${ }^{24}$ Strength and strength-endurance capacities were tested by bench press (1 repetition maximum (1-RM)) and pull-up count to exhaustion (maximum number of times a subject could pull up his own weight on a $2.5 \mathrm{~m}$ high cross bar). All subjects were familiar with the testing methods, since they were previously monitored regularly using these tests, which are the fitness tests prescribed by the United Cricket Board of South Africa (although no scientific study exists to prove their suitability for assessing cricket fitness). Blood samples were collected by venepuncture between $08 \mathrm{~h} 00$ and 09h00, after a 12-hour fast, no high-intensity exercise for 48 hours, and no exercise at all for 24 hours.

\section{Analysis}

EDTA blood samples were analysed for total and differential white blood cell (WBC) counts (Coulter STKS, Beckman/ Coulter, Fullerton, CA, USA), and heparin blood samples for distribution of lymphocyte subpopulations by three-colour flow cytometry (FACSCalibur, Becton/Dickinson, New Jersey, USA), using conjugated monoclonal antibodies (Multitest, Scientific Products, Johannesburg, SA). Activation of CD4+ and CD8+ T-cells was determined by measuring their expression of CD69 after in vitro exposure to a stimulant, using the method described earlier by Breytenbach et al. ${ }^{6}$ In short, aliquots of whole blood were stimulated for 4 hours $\left(37^{\circ} \mathrm{C}, 5 \% \mathrm{CO}_{2}, 98 \%\right.$ humidity $)$ in the presence of phorbol $12-$ myristate 13-acetate and a calcium ionophore, after which the cells were labelled with monoclonal antibodies specific for CD69 and measured using standard procedures for three-colour flow cytometry.

\section{Statistical analysis}

Results reported are means \pm standard deviation (SD). Data were compared using two-tailed, paired Student's $t$-tests and repeated measures analysis of variance (ANOVA) with Bonferroni post hoc tests. Associations between variables were determined using Pearson's correlation analysis. Significance level was set at $p<0.05$.

\section{Results}

Average body mass and height of subjects were $82.7 \pm 6.7$ $\mathrm{kg}$ and $1.81 \pm 0.07 \mathrm{~m}$ respectively. Prescribed training and match hours increased from 22 per week during the pre-season to 35 per week during the study (Table I). The main change in the components of training during this time were that weight training was removed during the season, and match-play hours, although intermittent due to the nature of the sport, increased from 6 to 24 hours. Exercise test results remained similar for the duration of the study (Table II).

Mean total WBC count did not change, but mean total lymphocyte count, as well as all lymphocyte subpopulation counts analysed, were significantly decreased (Table III). Specifically, CD4+ cells decreased by $21 \%$ and CD $8+$ cells by $16 \%$, with no significant change in the CD4:CD8 ratio. However, \% CD4+CD69+ cells increased significantly (B: $54.4 \pm 9.7 \%$, A: $64.0 \pm 8.5 \%, p<0.01$ ), while the $\%$ CD8+CD69+ cells remained similar (Fig. 1). The absolute number of cells activated per volume of blood did not change

TABLE II. Fitness performance of 14 professional male cricket players before and after intensive cricket participation lasting 5 weeks. No parameter changed significantly*

\begin{tabular}{lcc}
\hline Parameter & Before & After \\
\hline Bleep Test score & $12 \pm 1$ & $12 \pm 1$ \\
Estimated $\mathrm{VO}_{2 \max }(\mathrm{ml} / \mathrm{kg} / \mathrm{min})$ & 52.8 & 52.8 \\
Bench press 1-RM $(\mathrm{kg})$ & $84 \pm 10$ & $83 \pm 11$ \\
Bench press : weight ratio & $1.01 \pm 0.09$ & $1.01 \pm 0.09$ \\
Pull-up score & $11 \pm 4$ & $10 \pm 4$ \\
*Values are means \pm standard deviation. Data were compared using paired Student's \\
$t$-tests and significance was set at $p<0.05$. \\
1-RM = 1 repetition maximum; $\mathrm{VO}_{2 \text { max }}=$ maximal oxygen consumption.
\end{tabular}

\begin{tabular}{|c|c|c|c|c|}
\hline WBC subpopulations (cells/ul) & Normal range & Before & After & Significance \\
\hline Total WBC & $4000-11000$ & $6271 \pm 1333$ & $5607 \pm 1292$ & NS \\
\hline Total lymphocytes & $1000-4000$ & $2349 \pm 664$ & $1865 \pm 563$ & $p<0.005$ \\
\hline Total T-lymphocytes (CD3+) & $1100-1700$ & $1626 \pm 477$ & $1291 \pm 450$ & $p<0.005$ \\
\hline Helper T-lymphocytes (CD3+CD4+) & $700-1100$ & $949 \pm 316$ & $751 \pm 223$ & $p<0.01$ \\
\hline Suppressor/cytotoxic T-lymphocytes (CD3+CD8+) & $500-900$ & $550 \pm 196$ & $463 \pm 214$ & $p<0.05$ \\
\hline Ratio (CD3+CD4+:CD3+CD8+) & $1.5-2.0$ & $1.82 \pm 0.49$ & $1.79 \pm 0.53$ & NS \\
\hline NK cells (CD3+CD16+/ CD3+CD56+) & $200-400$ & $379 \pm 193$ & $266 \pm 152$ & $p<0.01$ \\
\hline B-lymphocytes (CD3+CD19+) & $200-400$ & $304 \pm 165$ & $246 \pm 103$ & $p<0.05$ \\
\hline
\end{tabular}


from $B$ to $A$ (Fig. 2).

The CD4:CD8 ratio correlated negatively with the Bleep Test score at both B and A (Fig. 3). At B, The Bleep Test score correlated negatively with \% CD4+CD69+ cells $(r=$ $-0.45, p<0.05)$ and tended to correlate negatively with $\%$ CD8+CD69+ cells $(r=-0.42, \mathrm{NS})$. Both these relationships no longer existed at $A$.

Six subjects each reported a short period (3 - 4 days) of URTS that cleared up with symptomatic treatment administered by the team doctor. Treatment consisted of either a nasal decongestant or oral anti-histamine, or both. One subject had diarrhoea that responded to an anti-diarrhoeal drug. None of the athletes discontinued training as a result of their symptoms. After dividing subjects into a symptomatic and an asymptomatic group, we found no effect of illness, and no interaction between illness and time (Table IV), although the significant effect of time on \% CD4+CD69+ cells was consistent with the data presented in Fig. 1.

\section{Discussion}

The main strengths of this study were firstly that it contributes to the few previous studies that have investigated the effect of participation in team sports on T-cell number and function ${ }^{7,25}$ secondly that the assessment of an activation marker on T-lymphocyte subpopulations was included in this study, and thirdly that the current results clearly indicate the importance of expressing immune function in absolute rather than in relative terms.

Previous longitudinal studies ${ }^{7,25}$ on football and soccer players showed that while the total WBC count remained unchanged, the CD4:CD8 ratio decreased over the course of a competitive season. The change in ratio was the result of an increase in CD8+ cells in the soccer players, ${ }^{25}$ but due to a decrease in the CD4+ cells in the football players. ${ }^{7}$ The results of unchanged total WBC count and decreased CD4+ cell count in the current study are in agreement with the study on footballers, ${ }^{7}$ whereas while the latter showed only a tendency for CD8+ cell count to decline, our study showed a significant decline. Our study does not support the finding of an increased CD8+ cell count reported in soccer players. ${ }^{25}$

The decreases in total lymphocyte, T-cell subpopulation, $B$ and NK cell counts reported here, are similar to results obtained in earlier studies of endurance athletes. ${ }^{1,27,32}$ It is known that exercise-induced increases in concentrations of stress hormones, such as catecholamines, ${ }^{8}$ may be responsible for the exercise-induced depression of the lymphocyte counts. ${ }^{3,9}$ Due to the nature of the game of cricket, all players are active for several hours each day when their side is bowling and fielding, and successful batsmen may also play for many hours on end, whilst those batsmen still to follow are under psychological stress. In cycling, the duration of exercise has been shown to be an important factor in altering the immune response. ${ }^{26}$ Although not all cricket players have equally long duration of exercise, and the exercise is different from that of cycling, the cumulative stress during a single match may play an important role in their immune response. The additive effect of several long cricket matches may have significant effects that are quite different from those that may be seen after a single bout of a typical high-intensity, inter-

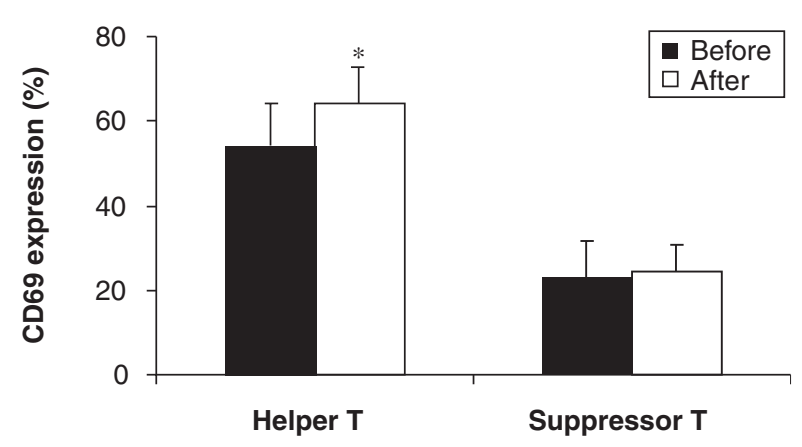

Fig. 1. Percentage T-lymphocytes expressing the CD69 antigen after in vitro stimulation before and after a strenuous professional cricket match schedule of 5 weeks' duration. Values are means $\pm S D$. (*Value after significantly different from value before $(p<0.01))$.

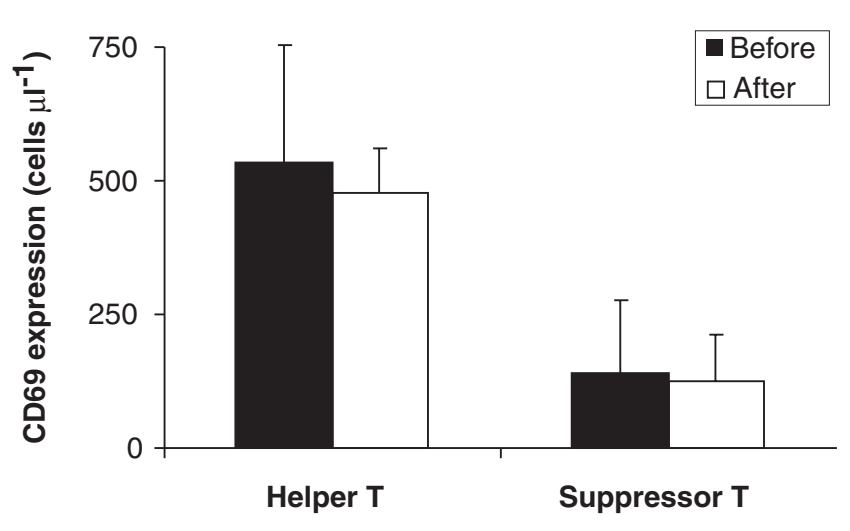

Fig. 2. Average absolute number of T-lymphocytes expressing the CD69 antigen after in vitro stimulation before and after a 5-week strenuous professional cricket matchschedule.

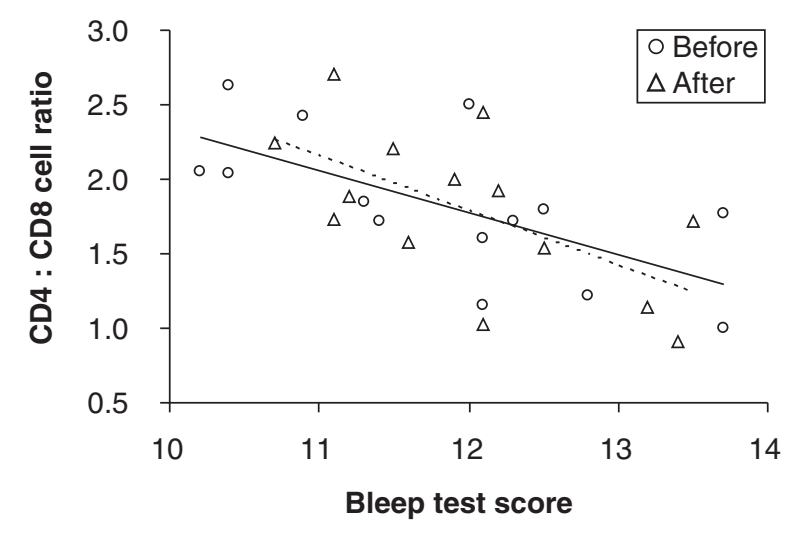

Fig. 3. The association between estimated $\mathrm{VO}_{2 \max }$ (from Bleep Test score) and helper:suppressorl cytotoxic $T$ cell ratio before $(0,--., r=-0.66, p<0.01)$ and after $(\Delta, \ldots, r=$ - 0.63, p < 0.01) the 5-week professional cricket matchschedule. 


\begin{tabular}{|c|c|c|c|c|c|c|c|}
\hline \multirow{3}{*}{$\frac{\text { Cell counts }}{\text { CD4+CD69+ (\%) }}$} & $\begin{array}{l}\text { Group 1: } \\
\text { URTS }\end{array}$ & $\begin{array}{l}\text { Group 2: } \\
\text { Healthy }\end{array}$ & $\begin{array}{l}\text { Group 1: } \\
\text { URTS }\end{array}$ & $\begin{array}{l}\text { Group 2: } \\
\text { Healthy }\end{array}$ & $\begin{array}{l}\text { Time } \\
\text { effect }\end{array}$ & $\begin{array}{r}\text { Group } \\
\text { effect }\end{array}$ & $\begin{array}{l}\text { Time-group } \\
\text { interaction }\end{array}$ \\
\hline & \multicolumn{2}{|c|}{ Time: Baseline } & \multicolumn{2}{|c|}{ Time: Follow-up } & \multicolumn{3}{|c|}{ Significance ( $p$-value) } \\
\hline & $53.5 \pm 9.0$ & $55.4 \pm 11.1$ & $66.6 \pm 6.1$ & $61.3 \pm 10.1$ & 0.01 & 0.67 & 0.31 \\
\hline CD8+CD69+ (\%) & $21.8 \pm 10.6$ & $24.1 \pm 6.8$ & $24.9 \pm 6.2$ & $23.7 \pm 6.9$ & 0.54 & 0.86 & 0.34 \\
\hline CD4+CD69+ (cells/ul) & $515 \pm 251$ & $549 \pm 207$ & $454 \pm 174$ & $499 \pm 94$ & 0.30 & 0.66 & 0.91 \\
\hline CD8+CD69+ (cells/ul) & $141 \pm 92$ & $136 \pm 80$ & $124 \pm 82$ & $122 \pm 99$ & 0.33 & 0.94 & 0.94 \\
\hline
\end{tabular}

mittent, short match duration team sport. Taking into consideration that the athletes in the current study also trained on days between matches, the periods of rest may have been insufficient for the immune system to recover from the combination of physical and mental stress endured during matches, and the physical stress of training. Exerciseinduced decreases in lymphocyte counts return to baseline levels only after 4 hours of recovery from acute exercise to exhaustion (lasting \pm 1 hour). ${ }^{25}$ We hypothesise that exercise frequency may affect total lymphocyte and lymphocyte subpopulation counts found at rest in competitive athletes due to repeated transient increases in stress hormone levels and insufficient recovery.

T-cell CD69 expression was also investigated. Although both CD4+ and CD8+ cell counts decreased proportionally (CD4:CD8 ratio stayed similar), the \%CD4+CD69+ on stimulation in vitro increased significantly. This is in contrast to the results of Ronsen et al. ${ }^{27}$ who showed no change in $\%$ CD4+CD69+ cells when determined directly after a second bout of exercise performed on the same day. Therefore, while a few acute bouts of exercise seem to have no effect on CD69 expression in CD4+ cells, many long-duration exercise bouts with additional cumulative stressors over 5 weeks resulted in significantly increased relative CD69 expression. Although acute exposure of immune cells to UV-B radiation in vitro decreased lymphocyte function, ${ }^{16}$ T-cell function was not decreased in our study. One reason for this discrepancy could be a difference in the magnitude of radiation during the in vitro experiment vs. during actual outdoor sport participation. Similar to extreme UV-B radiation, long-distance air travel also suppresses immune function. ${ }^{28,29}$ Since we showed an increase in lymphocyte function, it is unlikely that the shorter duration air travel experienced by our subjects was a confounding factor.

When considering the two main findings separately, the first finding of significantly decreased absolute counts of CD4+ and CD8+ cells at follow-up seems to indicate immune system impairment. But the second finding of an increased percentage of CD4+CD69+ cells seems to indicate that the immune system has an improved potential for functional response. The implication of both findings becomes clear only when put together, since the absolute number of activated $\mathrm{CD} 4+$ and $\mathrm{CD} 8+$ cells per volume of blood remained similar (CD4+CD69+ cells: B: $524 \pm 210$ vs. A: $487 \pm 130$ cells/ul; CD8+CD69+ cells: B: $136 \pm 78$ vs. A: $123 \pm 84$ cells/ul). Therefore, although circulating cell numbers decreased, the percentage of cells activated to undergo pro- liferation increased (Fig. 1), resulting in no net change in absolute cell reactivity (Fig. 2).

The Bleep Test score was negatively correlated with CD4+:CD8+ ratio at both $B$ and $A$, so that a higher score was associated with ratios favouring the non-specific/cytotoxic $T$ cell response, which is in accordance with previous reports in endurance athletes. ${ }^{18,19}$ In addition, the Bleep Test score correlated negatively with \% CD4+CD69+ cells $(r=-0.45, p$ $<0.05)$, and with \%CD8+CD69+ cells $(r=-0.42)$ before the competitive season. Therefore players with better performance in an endurance test had lower relative CD69 expression at the start of the study. These relationships were no longer evident at follow-up, possibly due to changes occurring during the match period, e.g. illness or a change in fitness levels. However, results failed to show differences in cell reactivity between subjects who had experienced periods of illness and and those who had not (Table IV), and fitness levels remained similar for the duration of the study (Table II). Other possible explanations include similarities in psychological stress and the additional stress of air travel between match venues during the study as opposed to the pre-season period.

The use of a suitable control group could have assisted in interpretation of data and strengthening of conclusions. However, selection of an appropriate control group would have been difficult for this study, and whether or not the investigators could control both their exercise habits and stress status for the duration of the study, in our case more than 2 months, is questionable. Such a group would have to consist of equally fit, age-matched, non-smoking subjects, with similar air travel and sun-exposure schedules, and without any changes in life stress for the duration of the study. Use of a non-suitable control group may also result in less accurate interpretation of results. For example, Bury et al. ${ }^{7}$ used non-trained students as a control group for their study. Such a group would be relevant for assessing exercise stress, but not any additive lifestyle stresses (such as examinations and social pressures). Indeed, their control group showed slightly decreased CD8+ cell count (4\%), which may have prevented the lowered CD8+ cell count seen in the footballers $(15 \%)$ from reaching statistical significance, despite being of similar magnitude to the decline seen in CD8+ cell count in the cricket players in our study (16\%). Therefore, in the current study a control group was not used, and baseline samples served as controls, similar to other previous studies of long duration investigating similar parameters. ${ }^{10,25}$ 


\section{Conclusion}

This study has shown that a 5-week strenuous, team sport match schedule had no net suppressive effect on CD4+ and CD8+ cell reactivity in response to mitogen stimulation, despite decreased circulating numbers of CD4+ and CD8+ cells. Therefore, it is concluded that assessing absolute CD4+ and CD8+ cell counts may lead to incorrect conclusions about athletes' immune systems. It is proposed that potential capacity for lymphocyte function should be assessed by determining the absolute number of cells per volume of blood expressing CD69.

\section{Acknowledgements}

This study was funded by the South African Medical Research Council.

\section{RefERENCES}

1. Baj Z, Kantorski J, Majewska E, et al. Immunological status of competitive cyclists before and after the training season. Int J Sports Med 1994; 15 319-24.

2. Berglund $B$, Hemmingsson P. Infectious disease in elite cross-country skiers: a one-year incidence study. Clin Sports Med 1990; 2: 19-23.

3. Berk LS, Nieman DC, Youngberg WS, et al. The effect of long endurance running on natural killer cells in marathoners. Med Sci Sports Exerc 1990 21: 207-12.

4. Borrego F, Alonso MC, Galiani MD, et al. NK phenotypic markers and IL2 response in NK cells from elderly people. Exp Gerontol 1999a; 34: 25365.

5. Borrego F, Robertson MJ, Ritz J, Pena J, Solana R. CD69 is a stimulatory receptor for natural killer cells and its cytotoxic effect is blocked by CD94 inhibitory receptor. Immunology 1999b; 97: 159-65.

6. Breytenbach $\mathrm{U}$, Clark A, Lamprecht J, Bouic P. Flow cytometric analysis of the Th1-Th2 balance in healthy individuals and patients infected with the human immunodeficiency virus (HIV) receiving a plant sterol/sterolin mixture. Cell Biol Int 2001; 25(1): 43 - 9

7. Bury T, Marechal R, Mahieu P, Pirnay F. Immunological status of competitive football players during the training season. Int J Sports Med 1998; 19: 364-8.

8. Carlson SL, Beiting DJ, Kiani CA Abell KM, McGillis JP Catecholamines decrease lymphocyte adhesion to cytokine-activated endothelial cells. Brain Behav Immun 1996; 10: 55-67.

9. Fry RW, Morton AR, Crawford GPM, Keast D. Cell numbers and in vitro responses of leukocytes and lymphocyte subpopulations following maximal exercise and interval training sessions of different intensities. Int $J$ Sports Med 1992; 13: 359-65.

10. Gabriel H, Schmitt B, Urhausen A, Kindermann W. Increased CD45RA+CD45RO+ cells indicate activated T cells after endurance exercise. Med Sci Sports Exerc 1993; 25: 1352-7.

11. Gleeson M, McDonald WA, Pyne DB, et al. Immune status and respiratory illness in elite swimmers during a 12-week training cycle. Int $J$ Sports Med 2000; 21: 302-7.

12. Green KJ, Rowbottom DG, Mackinnon LT. Acute exercise and T-lymphocyte expression of the early activation marker CD69. Med Sci Sports Exerc 2003; 35: 582 - 8.
13. Mackinnon LT. Overtraining effects on immunity and performance in athletes. Immunol Cell Biol 2000; 78: 502-9.

14. Mardiney M, Brown MR, Fleisher TA. Measurement of T-cell CD69 expression: a rapid and efficient means to assess mitogen- or antigen-induced proliferative capacity in normals. Cytometry (Communications in Clinical Cytometry) 1996; 26: 305-10.

15. Moller $\mathrm{P}$, Wallin $\mathrm{H}$, Holst $E$, Knudsen LE. Sunlight-induced DNA damage in human mononuclear cells. FASEB J 2002; 16(1): 45 - 53.

16. Morison WL, Parrish JA, Bloch KJ, Krugler JI. In vivo effect of UV-B on lymphocyte function. Br J Dermatol 1979; 101: 513 - 9.

17. Mueller O, Villiger B, O'Callaghan B, Simon HU. Immunological effects of competitive versus recreational sports in cross-country skiing. Int J Sports Med 2001; 22: 52-9.

18. Natale VM, Brenner IK, Moldoveanu AI, Vasiliou P, Shek P, Shephard RJ. Effects of three different types of exercise on blood leukocyte count during and following exercise. Sau Paulo Medical Journal 2003; 121(1): 9-14.

19. Nielsen HB. Lymphoycte responses to maximal exercise: a physiological perspective. Sports Med 2003; 33: 853-67.

20. Nieman DC. Current perspective on exercise immunology. Current Sports Medicine Reports 2003; 2: 239 - 42.

21. Nieman DC. Is infection risk linked to exercise workload? Med Sci Sports Exerc 2000; 32: S406-S411.

22. Nieman DC, Pederson BK. Exercise and immune function. Sports Med 1999; 27(2): 73-80,

23. Peters EM, Bateman ED. Ultramarathon running and upper respiratory tract infections - an epidemiological survey. S Afr Med J 1983; 64: 582-4.

24. Ramsbottom R, Brewer J, Williams $C$. A progressive shuttle run test to estimate oxygen uptake. Br J Sports Med 1988; 22: 141-4.

25. Rebelo AN, Candeias JR, Fraga MM, et al. The impact of soccer training on the immune system. J Sports Med Phys Fitness 1998; 38: 258 - 61.

26. Robson PJ, Blannin AK, Walsh NP, Castell LM, Gleeson M. Effects of exercise intensity, duration and recovery on in vitro neutrophil function in trained-male cyclists. Int J Sports Med 1999; 20: 128-35.

27. Ronsen O, Pedersen BK, Ortisland TR, Bahr R, Kjeldsekragh J. Leukocyte counts and lymphocyte responsiveness associated with repeated bouts of strenuous endurance exercise. J Appl Physiol 2001; 91: 425-34.

28. Rose DM, Jung D, Tamm W, Keth A, Loos AH. Changes in cellular proliferation rate of lymphocytes after long-distance flights as a possible risk for patients with HIV-infection. Eur J Med Res 2000; 5: 411 - 4.

29. Rose DM, Jung D, Parera D, Koniettzko J. Time zone shift and the immune system during long-distance flights (Abstract only). Z Arztl Fortbild Qualitatssich 1999; 93: 481 - 4.

30. Shek PN, Sabiston BH, Buguet A, Radomski MW. Strenuous exercise and immunological changes: a multi-time-point analysis of leukocyte subsets CD4:CD8 ratio, immunoglobulin production and the NK cell response. Int J Sports Med 1995; 16: 446-74.

31. Shephard RJ. Overview of the epidemiology of exercise immunology. Immunol Cell Biol 2000; 78: 485-95.

32. Shephard RJ, Shek PN. Exercise and CD4+/CD8+ cell counts: influence of various contributing factors in health and HIV infection. Exerc Immunol Rev 1996; 2: 65-83.

33. Shephard RJ, Shek PN. Infectious diseases in athletes: New interest for an old problem. J Sports Med Phys Fitness 1994; 34: 11-22.

34. Smith LL. Overtraining, excessive exercise, and altered immunity: Is this a T helper-1 versus T helper-2 lymphocyte response? Sports Med 2003; 33 $347-64$.

35. Werfel T, Boeker M, Kapp A. Rapid expression of the CD69 antigen on T-cells and natural killer cells upon antigenic stimulation of periphera blood mononuclear cell suspensions. Allergy 1997; 52: 465-9. 